\title{
Isolated central hypoadrenalism as the sole manifestation of
}

presumed neurosarcoidosis

Sviatlana Zhyzhneuskaya, Jehangir Abbas, Murali Ganguri, Sath Nag Department of Endocrinology,

The James Cook University Hospital, Middlesbrough
South Tees Hospitals WHS

NHS Foundation Trust

\section{Introduction:}

- Hypothalamic-pituitary sarcoidosis is uncommon and affects less than $10 \%$ of patients with neurosarcoidosis.

- It presents a diagnostic challenge.

-We present a case of isolated central hypoadrenalism as the sole manifestation of neurosarcoidosis

\section{Case History:}

-76 year old man with recently diagnosed primary hypothyroidism -Presented with weight loss, lethargy and fatigue

-Physical exam : inguinal lymhadenopathy

-Staging CT thorax: bilateral mediastinal lymphadenopathy,

(Picture 1) interstitial lung parenchymal changes, and splenomegaly

-Mild hypercalcaemia (Calcium-2.65 $\mathrm{mmol} / \mathrm{l}$ ) and hyponatremia noted - Initial DD:lymphoma, malignancy, or granulomatous disease

\section{Investigations:}

-Diagnostic excision biopsies of left inguinal and mediastinal lymph nodes were inconclusive -ACTH stimulation with tetracosactide: sub-optimal cortisol increment with peak of $224 \mathrm{nmol} / /$ - Adrenal antibodies were negative -Serum ACTH <5 mg/l

- Secondary adrenal insufficiency confirmed by a subsequent long ACTH stimulation test (Table 1) -CT of pituitary gland: no gross abnormality

\section{Case Progress:}

-An empirical trial of steroid therapy for presumed sarcoidosis commenced with symptomatic improvement

-Withdrawal of steroids attempted in view of the inconclusive lymph node biopsies which resulted in a marked deterioration with hypotension, listlessness and obtundation

-Adrenal insufficiency was suspected

- Improved symptomatically after reinitiating glucocorticoids

Table 1

Thoracic CT

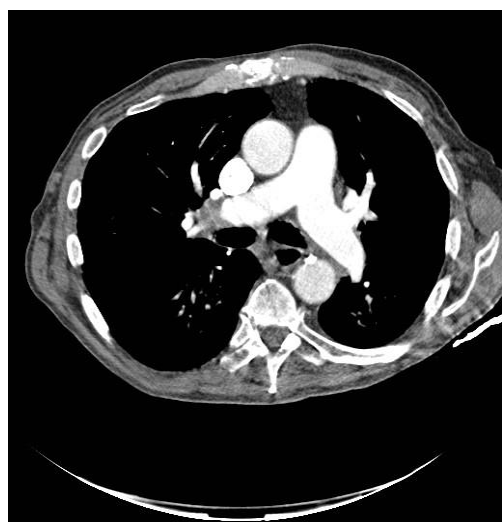

Long Synacthen Test

\begin{tabular}{|l|l|}
\hline Time & $\begin{array}{l}\text { Cortisol } \\
(\mathrm{nmol} / \mathrm{l})\end{array}$ \\
\hline Baseline & $<28$ \\
\hline 8 Hours & 414 \\
\hline 24 Hours & 1024 \\
\hline
\end{tabular}

\section{Discussion:}

-The exact mechanism of injury in hypothalamicpituitary sarcoidosis is unclear -Endocrine dysfunction was historically attributed to granulomatous infiltration of the hypothalamus and pituitary though hypothalamic insufficiency and pituitary responsiveness to hypothalamic releasing factors are now thought to be the main cause of hypopituitarism

- Central hypoadrenalism can present with protracted and non-specific symptoms and pose a diagnostic challenge in the absence of any gross structural pituitary abnormality -A high index of suspicion remains the cornerstone of diagnosis 